
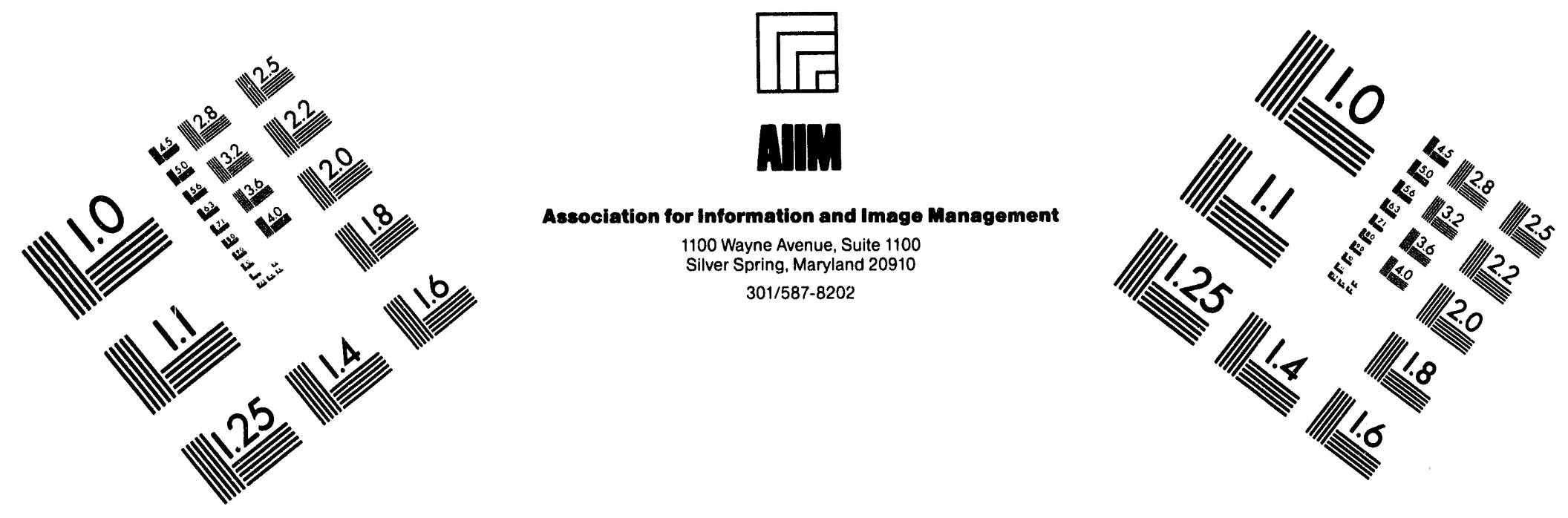

\title{
Centimeter
}

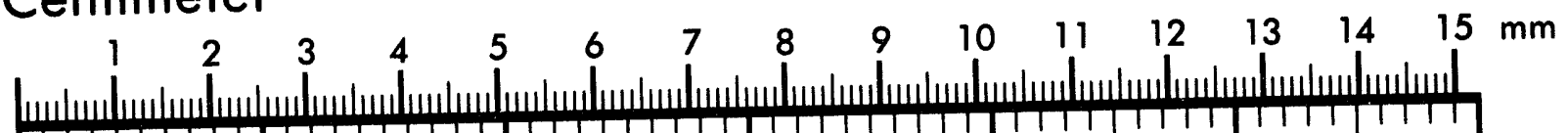

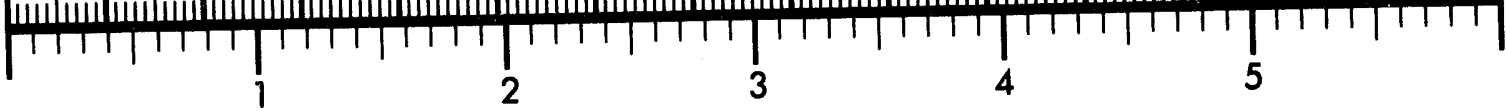
Inches
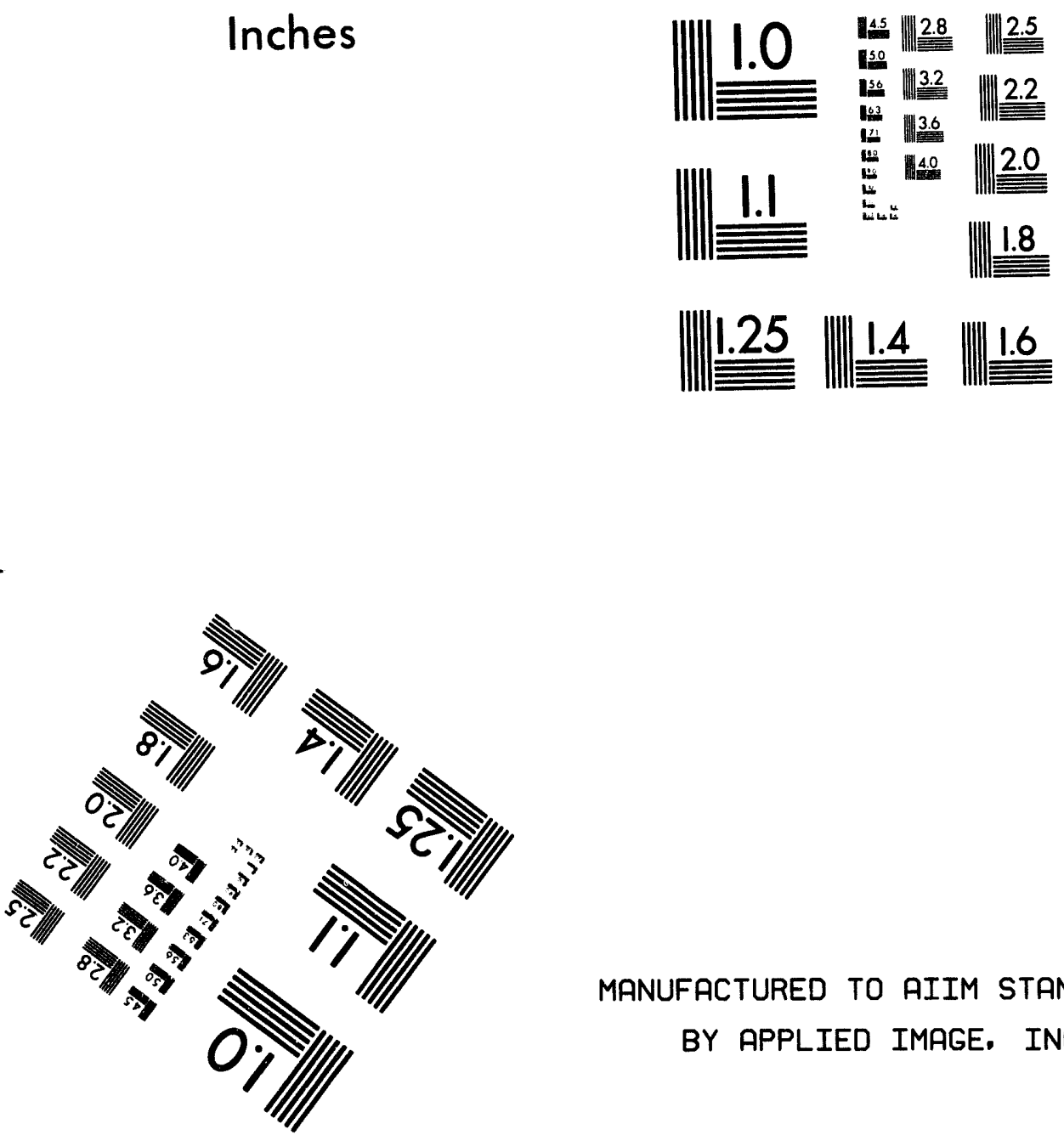

MANUFACTURED TO AIIM STANDARDS

BY APPLIED IMAGE, INC.

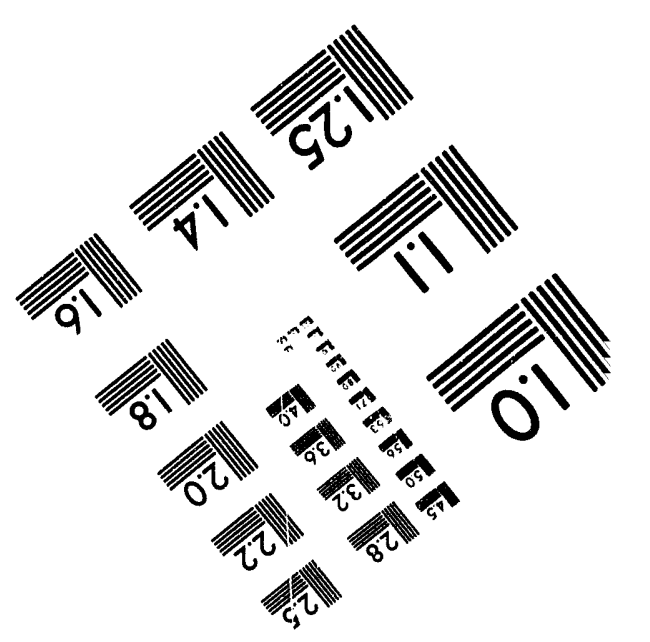



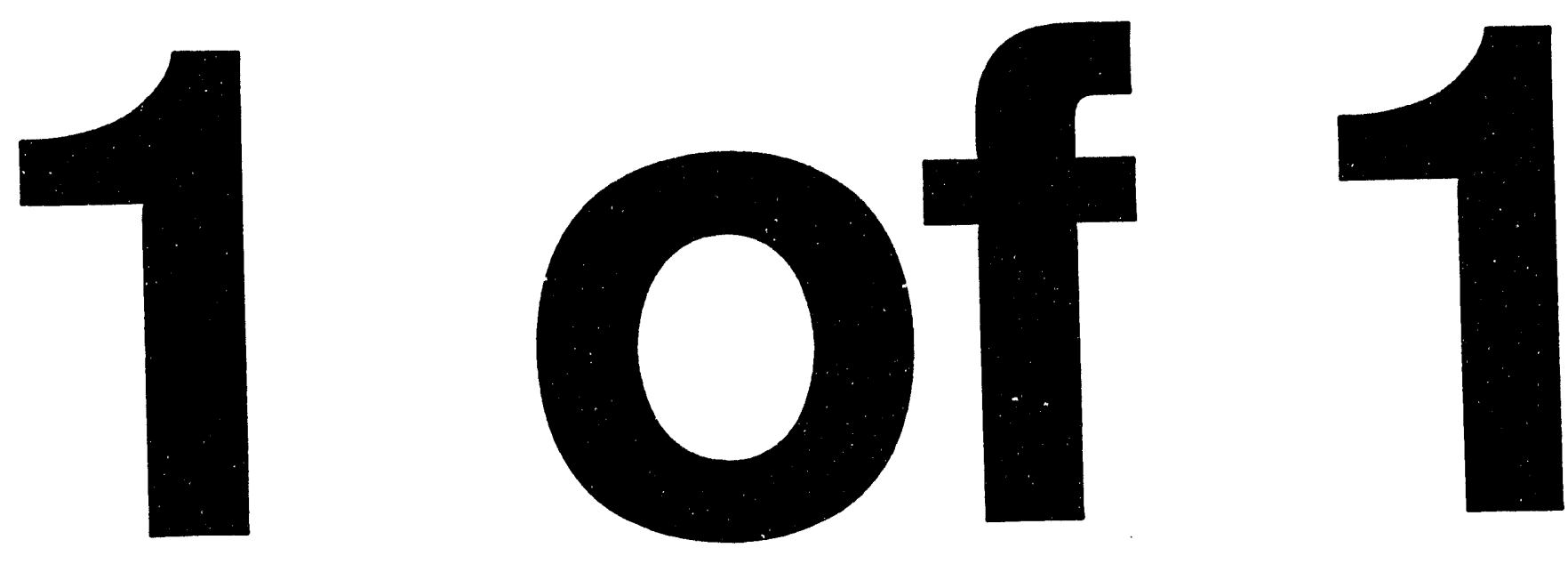


\section{La-ur- $94-1588$}

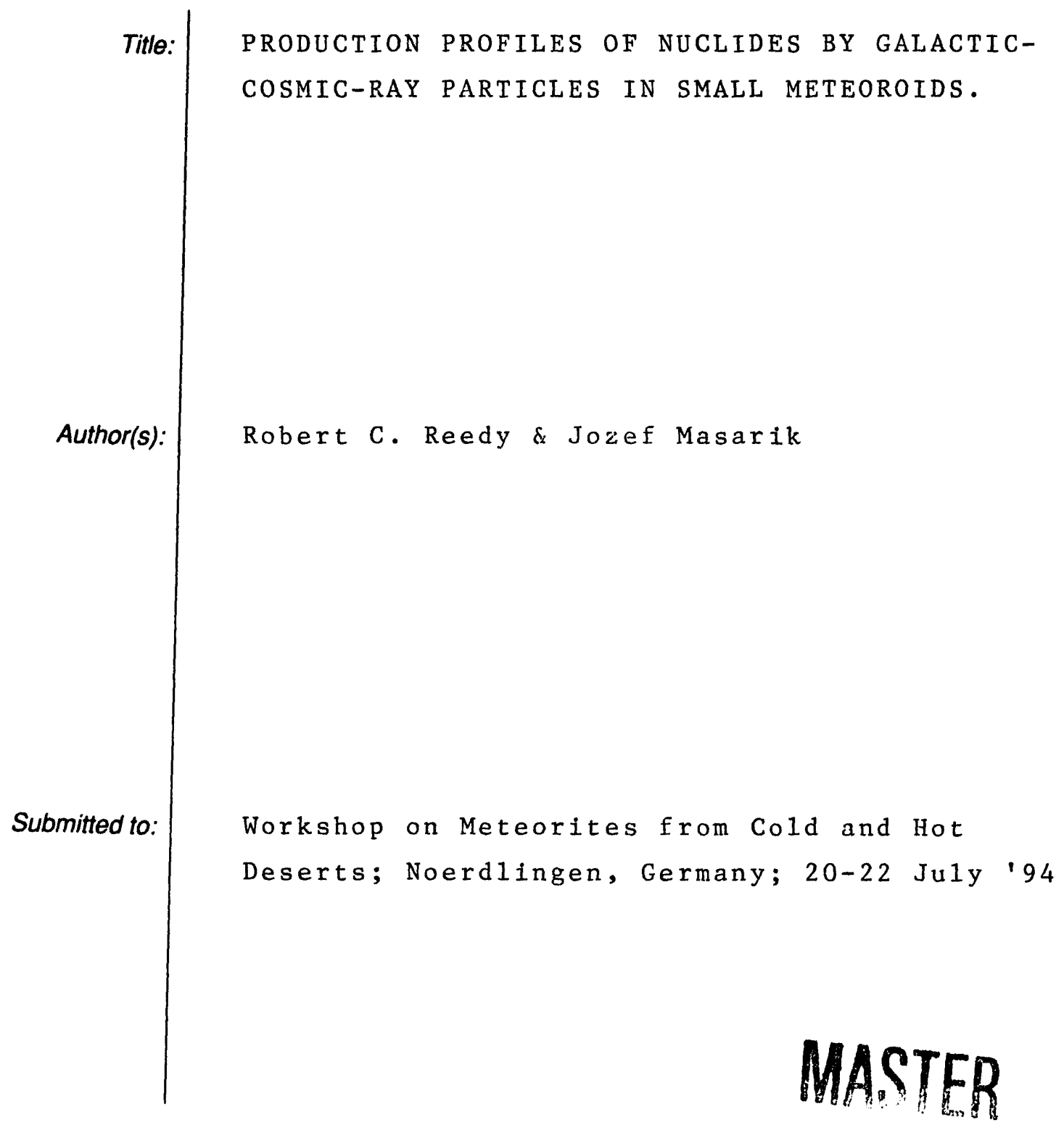

\section{Los Alamos WATIONAL LABOAATOAY}

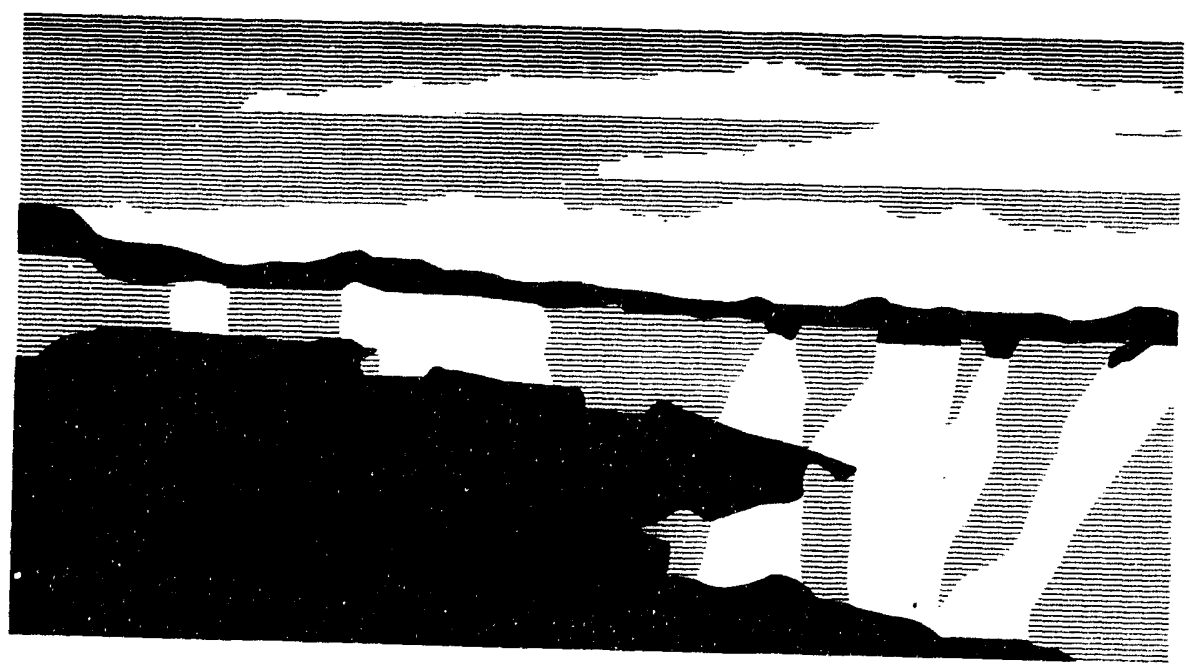

Los Alamos National Laboratory, an affirmative action/equal opportunity employer, is operated by the University of California tor the $U$. publish or reproduce the published form of this of this article, the publisher recognizes that the U.S. Government retains a nonexclusive, royalty-free license to requests that the publisher identify this articte as work performed under the auspices of the U.S. Department purposes. The Los Alamos National Laboratory

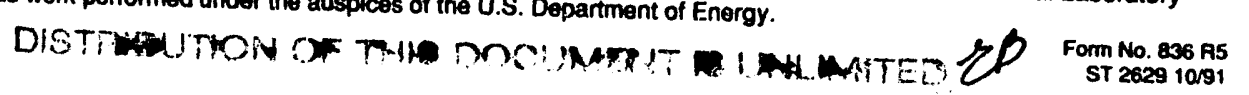


PRODUCTION PROFILES OF NUCLIDES BY GALACTIC-COSMICRAY PARTICLES IN SMALL METEOROIDS.* R. C. Reedy and J. Masarik, Los Alamos National Laboratory, Los Alamos, NM 87545, USA.

Many of the meteorites found in cold and hot deserts are small, and many were small bodies in space. Production of cosmic-ray-produced (cosmogenic) nuclides in small meteoroids is expected to be different than that in the larger meteoroids typically studied [1], with lower levels of nuclide production by galactic-cosmic-ray (GCR) particles [e.g., 2] and possibly significant production by solar-cosmic-ray (SCR) protons [3]. Motivated by the cosmogenic-nuclide measurements for the very small Salem meteorite $[4,5]$ and for cosmic spherules [e.g., 6], which show high levels of SCR production, we have reported earlier nuclide production rates by SCR protons in small objects in space [e.g., 3,7]. The GCR production rates reported in [2] for small meteoroids have not been tested and were expected to be poor for meteoroids with radii $<40 \mathrm{~g} / \mathrm{cm}^{2}$ because of the very simple nature of that semi-empirical model (only one free parameter) and because the mix of neutrons and protons is different (relatively more protons) than that in the model, which was based on larger objects. Thus we have calculated production rates for nuclides made by GCR particles in small objects with a physical model that is much better suited for unusual targets.

The production rates for GCR nuclides were calculated using particle fluxes from the Los Alamos Monte Carlo LAHET Code System (LCS) and measured or evaluated cross sections [8]. LCS has yielded calculated production rates that almost always are in very good agreement with cosmogenic-nuclide measurements in meteorites with radii greater than about $15 \mathrm{~cm}[8,9]$. The fluxes of protons and neutrons in spherical meteoroids of radii from 1 to $45 \mathrm{~cm}$ and with an L-chondrite composition were calculated as a function of pre-atmospheric depth. Production rates for most stony objects are similar to those for L-chondrites [8]. The neutron and protons fluxes calculated by LCS were normalized to an effective incident omnidirectional GCR proton flux of 4.8 protons $/\left(\mathrm{cm}^{2} \mathrm{~s}\right)$ [9]. This flux is $\sim 60 \%$ higher than that for the primary protons in the GCR as it includes production by alpha particles and heavier GCR nuclei, both directly and by secondary nucleons contributed by the break-up or reactions of these heavier GCR nuclei. We have found that the GCR flux averaged over the orbits of meteoroids are $~ 5 \%$ greater than that at the Moon [10]. For each layer, these fluxes were then multiplied by the neutron and proton cross sections for major target elements and integrated over energy to get the production rates for eight nuclides: ${ }^{10} \mathrm{Be},{ }^{14} \mathrm{C},{ }^{21} \mathrm{Ne},{ }^{22} \mathrm{Ne},{ }^{26} \mathrm{Al},{ }^{36} \mathrm{Cl},{ }^{38} \mathrm{Ar}$, and ${ }^{53} \mathrm{Mn}$.

The calculated GCR production rates for the smallest meteoroids are $~ 70 \%$ greater than those calculated using only primary GCR protons by [11], mainly because of the $\sim 60 \%$ factor for heavier GCR nuclei, which do not break-up or react in very small meteoroids. Our calculated GCR production rates for radii up to $\sim 10 \mathrm{~cm}$ also could be high. The only test of GCR production rates in very small meteoroids is the ${ }^{10} \mathrm{Be}$ activities in Salem, which are $\approx 17 \mathrm{dpm} / \mathrm{kg}$, in good agreement with our ${ }^{10} \mathrm{Be}$ production rates calculated for a 3 -cm-radius meteoroid. Thus the most serious errors in our calculated production rates are for radii less than $\sim 3 \mathrm{~cm}$. We are making additional LCS calculations using incident GCR alpha particles to test directly the contributions of heavier GCR nuclei in small objects.

The plots in Fig. 1 show the results of our production-rate calculations. All of the cosmogenic nuclides have GCR production profiles that increase from the pre-atmospheric surface to a maximum near the center of the meteoroid. The results are basically similar to those in [2]. The amount of this increase varies considerably, with products made by higher-energy particles and by protons, such as ${ }^{10} \mathrm{Be}$, having less increase in production rate with depth. In all cases, the GCR-calculated production rates in meteoroids with radii less than $\sim 15 \mathrm{~cm}$ are much less than those for larger meteoroids. As most cosmogenicnuclide production rates have been inferred from measured concentrations in such larger meteoroids, their use for small meteoroids results in over-estimated production rates. For 
example, our calculated ${ }^{21} \mathrm{Ne}$ prcduction rate for Salem as a 3-cm sphere and the measured ${ }^{21} \mathrm{Ne}$ concentration [12] yields an exposure age for Salem of about $15 \mathrm{Ma}$, more than the 9.5$\mathrm{Ma}$ age reported in [5] using systematics based on larger objects, although our calculated ${ }^{22} \mathrm{Ne} /{ }^{21} \mathrm{Ne}$ ratio $(\sim 1.35)$ is higher than the observed ratio of 1.23 . However, this is an upper limit to the age as SCR contributions were not included. Also, possible complications, such as erosion in space [13], have also not been considered.

Except probably for ${ }^{10} \mathrm{Be}$, which has quite low SCR-production rates [14], it should be remembered that nuclide production by SCR particles should be added to the GCR contribution in interpreting measured concentrations of cosmogenic nuclides. These SCR contributions can be significant, especially near the pre-atmospheric surface and in the imaller meteoroids $[3,7]$. The amount of the SCR contribution is not only sensitive to the pre-atmospheric radius of the meteorite and a sample's pre-atmospheric depth $[3,7]$, but also to the orbit of the meteoroid prior to hitting the Earth. The time-averaged flux of SCR particles decreases rapidly with increasing distance from the Sun, as $R^{-2}$ because of the distance from the Sun and possibly with poorly-known additional factors of up to $R^{-0.5}$ or more due to interactions of SCR particles with the interplanetary mediurn [15].

References: [1] Reedy R.C. (1990) Meteoritics 25, 400. [2] Reedy R.C. (1985) PLPSC15th, C722. [3] Reedy R.C. (1987) LPS XVIII, 822. [4] Evans J.C. et al. (1987) LPS XVIII, 271. [5] Nishiizumi K. et al. (1990) Meteoritics 25, 392. [6] Nishiizumi K. et al. (1990) EPSL 104, 315. [7] Reedy R.C. (1990) LPS XXI, 1001. [8] Masarik J. and Reedy R. C. (1994) GCA, in press. [9] Reedy R.C. et al. (1993) LPS XXIV, 1195. [10] Reedy R.C. and Masarik J. (1994) LPS XXV, 1119. [11] Reedy R.C. (1987) PLPSC-17th, E697. [12] Wieler R. (1992) Priv. Comm. [13] Reedy R.C. (1992) Meteoritics 27, 280. [14] Nishiizumi K. et al. (1988) PLPSC-18th, 79. [15] Hamilton D.C. (1988), In JPL Publ. 88-28, p. 86. * Work supported by NASA and done under the auspices of the U.S. Dept. of Energy.
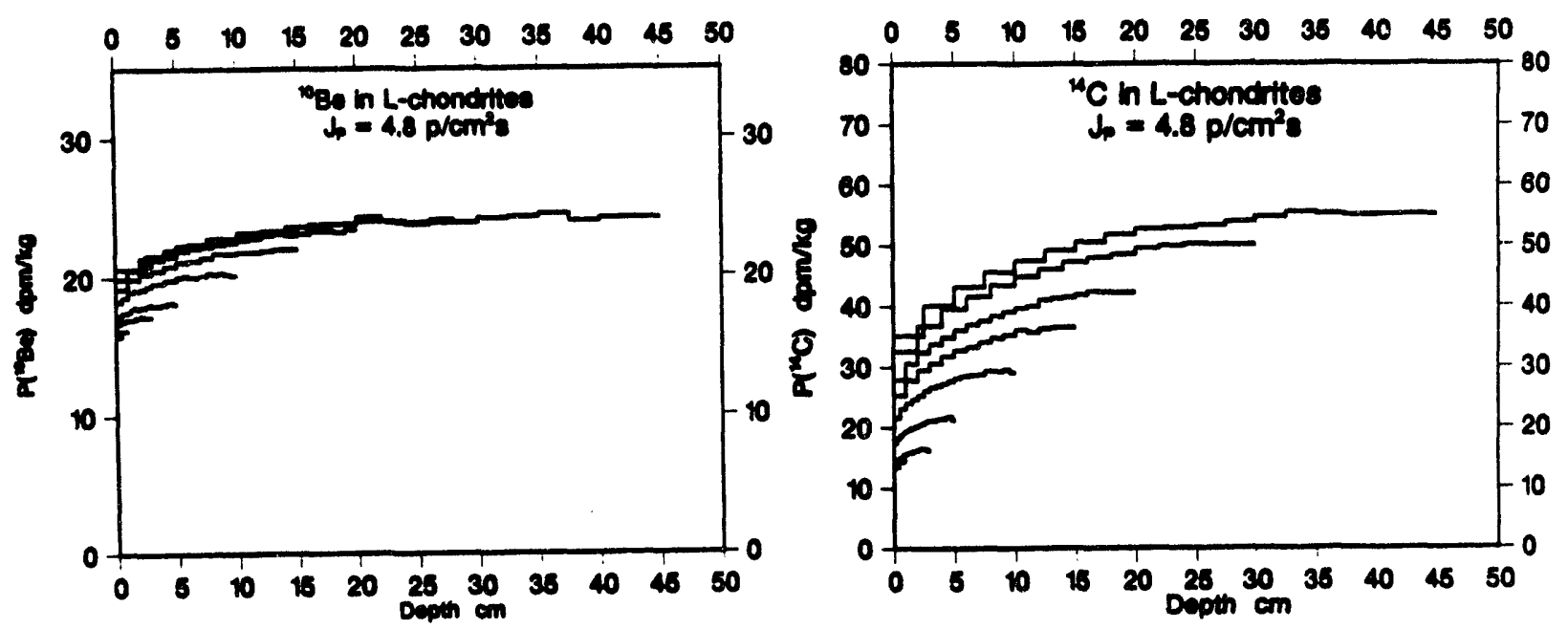

Figure 1. Calculated GCR production rates (or ratios) as a function of depth in meteoroids of various pre-atmospheric radii. 

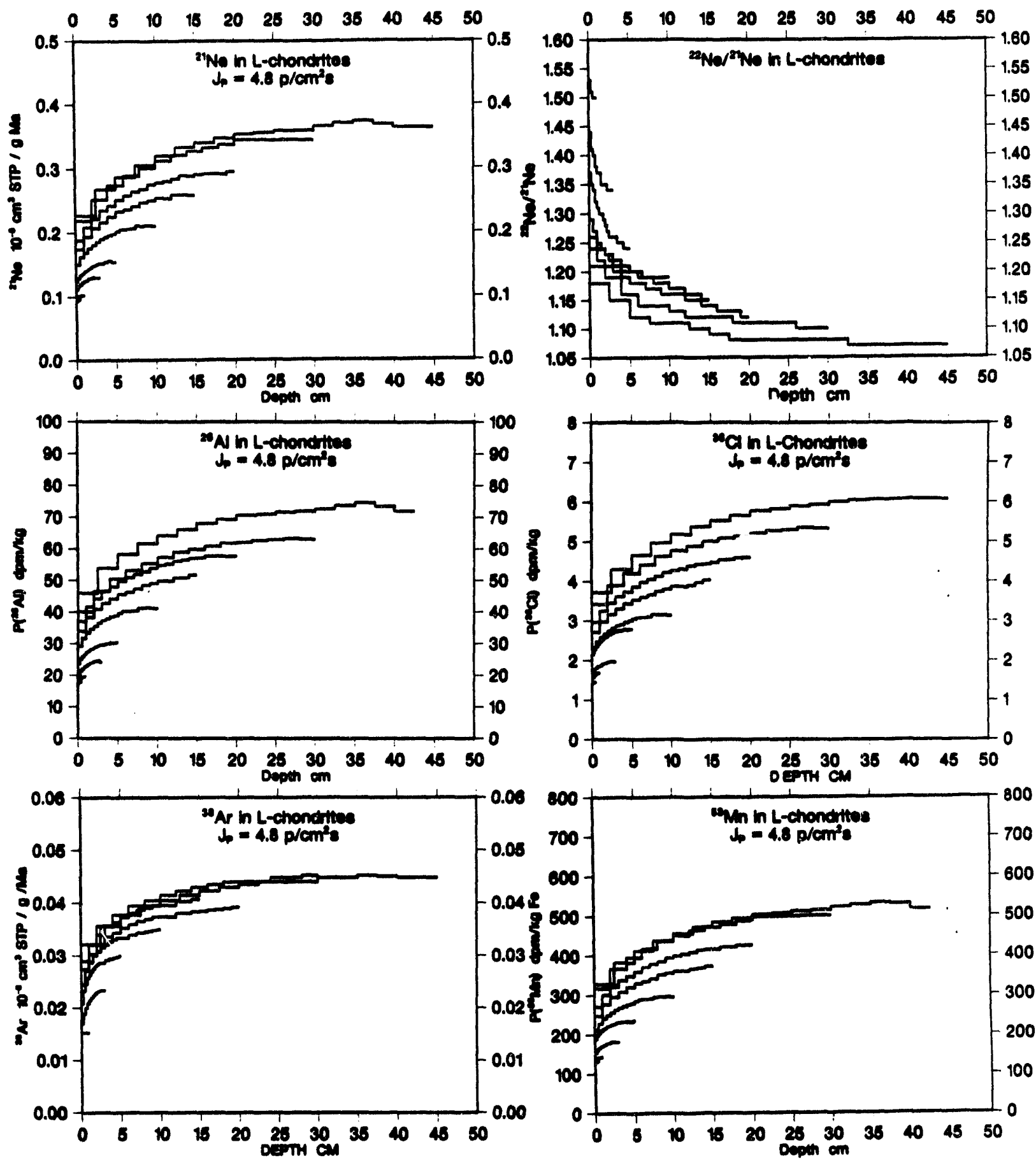

Figure 1, cont. Calculated GCR production rates (or ratios) as a function of depth in meteoroids of various pre-atmospheric radii. 


\section{DATE}
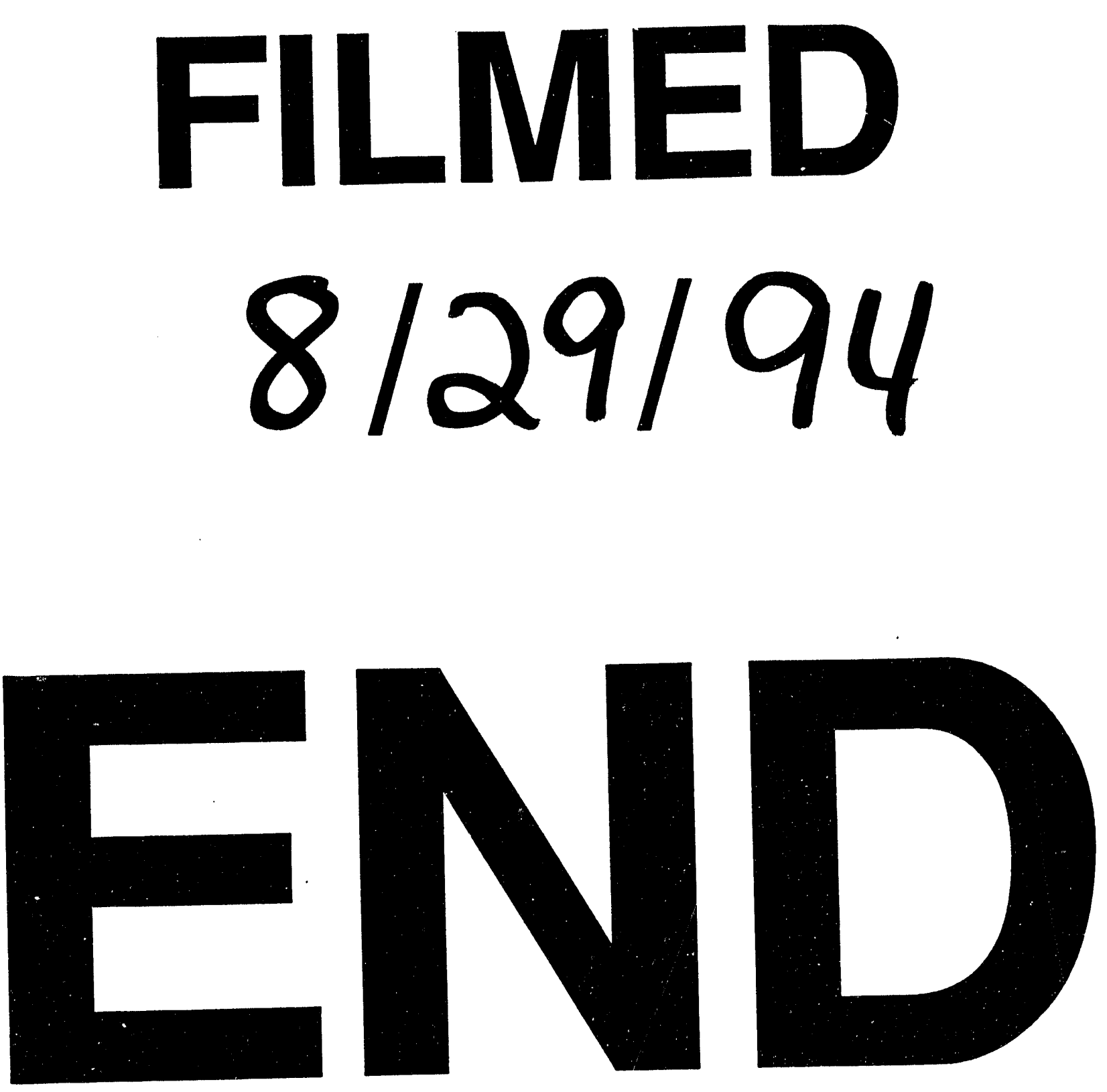
